\title{
Identidades profesionales de extensionistas rurales en diferentes países de África, América Latina y Oceanía: un estudio comparativo
}

\section{Professional identities of rural extension workers in different countries of Africa, Latin America and Australia: a comparative study}

Fernando Landini'; Sandra Hoffman-Martins'; Manuela Martinez ${ }^{3}$; Eduardo Tarantini'; Carlos Nische ${ }^{5}$; Haydelín Rodríguez-Chávez ${ }^{6}$

'Lic. en Psicología, M.Sc., Ph.D. Universidad de la Cuenca del Plata, UCP; Consejo Nacional de Investigaciones Científicas y Técnicas, CONICET; Misiones, Argentina; e-mail: landini_fer@hotmail.com; (1) https://orcid.org/0000-0002-5322-2921

Lic. en Psicología. Estudiante de doctorado. Universidad de la Cuenca del Plata, UCP; Consejo Nacional de Investigaciones Científicas y Técnicas, CONICET; Misiones, Argentina; e-mail: martinssand@hotmail.com; (D) https://orcid.org/0000-0003-0853-6590

${ }^{3}$ Lic. en Psicología. Estudiante de doctorado. Universidad de la Cuenca del Plata, UCP; Consejo Nacional de Investigaciones Científicas y Técnicas, CONICET; Misiones, Argentina; e-mail: manuelamartinez.r@outlook.com.ar; (iD https://orcid.org/0000-0002-4863-4411

${ }^{4}$ Lic. en Psicología. Estudiante de doctorado. Universidad de la Cuenca del Plata, UCP; Consejo Nacional de Investigaciones Cientíícas y Técnicas, CONICET; Misiones, Argentina; e-mail: edu_tarantini@hotmail.com; (D) https://orcid.org/0000-0003-0457-8419

${ }^{5}$ Lic. en Psicología. Estudiante de doctorado. Universidad de la Cuenca del Plata, UCP; Consejo Nacional de Investigaciones Científicas y Técnicas, CONICET; Misiones, Argentina; e-mail: maurycmn@hotmail.com; (D) https://orcid.org/0000-0001-5725-4202

${ }^{6}$ Lic. en Psicología. Estudiante de doctorado. Universidad de la Cuenca del Plata, UCP; Consejo Nacional de Investigaciones Científicas y Técnicas, CONICET; Misiones, Argentina; e-mail: hrodchavez@gmail.com; (D) https://orcid.org/0000-0002-1747-5833

Cómo citar: Landini, F.; Hoffman-Martins, S.; Martinez, M.; Tarantini, E.; Nische, C.; Rodríguez-Chávez, H. 2021. Identidades profesionales de extensionistas rurales en diferentes países de África, América Latina y Oceanía: un estudio comparativo. Rev. U.D.C.A Act. \& Div. Cient. 24(1):e1914. http://doi.org/10.31910/rudca.v24.n1.2021.1914

Artículo de acceso abierto publicado por Revista U.D.C.A Actualidad \& Divulgación Científica, bajo una licencia Creative Commons CC BY-NC 4.0

Publicación oficial de la Universidad de Ciencias Aplicadas y Ambientales U.D.C.A, Institución de Educación Superior Acreditada de Alta Calidad por el Ministerio de Educación Nacional.

Recibido: mayo 1 de 2020 Aceptado: abril 9 de $2021 \quad$ Editado por: Helber Adrián Arévalo-Maldonado

\section{RESUMEN}

La extensión rural es una profesión altamente compleja. Es claro que las prácticas más efectivas, se deben adaptar de manera flexible a los contextos y no se pueden pensar en términos de la aplicación tecnocrática de procedimientos. Las identidades profesionales organizan el modo en que los integrantes de diferentes profesiones comprenden su rol y dan forma a sus prácticas laborales. En este trabajo, se estudian las identidades profesionales de los extensionistas, a fin de contribuir a la comprensión de sus prácticas. A partir de un cuestionario, se analizan las identidades profesionales de extensionistas rurales de Argentina, Australia, Brasil, Chile, México, Nueva Zelanda, Nigeria, Paraguay y Sudáfrica. Los datos fueron procesados con el apoyo del software SPSS. Partiendo de una 
tipología teórica, se observa que la identidad más frecuente es la de un profesional orientado al apoyo productivo-empresarial, seguida por la de aquellos que piensan su rol en términos de mejora de la calidad de vida de los productores. Son pocos los que conciben su profesión en términos de impulsar procesos de cambio social y superación de situaciones de injusticia. Se diferencian dos tipos de países, aquellos donde predomina la identidad de profesionales que apoyan a los agricultores, a nivel productivo-empresarial y aquellos donde esta identidad posee un porcentaje similar a la centrada en la mejora de la calidad de vida. En Nigeria y Sudáfrica es más frecuente la presencia de extensionistas, que se conciben como impulsores de procesos de cambio social.

Palabras clave: Identidad profesional; Extensión rural; Productividad agrícola; Calidad de vida; Cambio social.

\section{ABSTRACT}

Rural extension is a highly complex profession. It is apparent that the most effective practices have to adapt flexibly to the contexts, and cannot be understood in terms of the application of technocratic procedures. Professional identities organize how practitioners understand their role and shape their job practices. In this article, the professional identities of extension workers are studied, with the aim to contribute to the understanding of their practices. Based on a questionnaire, the professional identities of extension workers from Argentina, Australia, Brazil, Chile, Mexico, New Zealand, Nigeria, Paraguay and South Africa were analyzed. Data was processed using SPSS software. Using a theoretical typology, results show that the most frequent identity is that of a professional aimed at supporting farmers at a productive or business level, followed by those who understand their role in terms of improving farmers' quality of life. Finally, only few respondents conceive their role as promoters of processes of social change and overcoming of injustice situations. Two types of countries were identified, one where the identity of supporting farmers at a productive or business level prevails, and other where this identity has similar support to that of helping farmers to improve their quality of life. In Nigeria and South Africa, it is more frequent to find extension workers who understand themselves as facilitators of processes of social change.

Keywords: Professional identity; Rural extension; Agricultural productivity; Quality of life; Social change.

\section{INTRODUCCIÓN}

Existe un creciente interés en la literatura académica por el estudio de las identidades de profesionales de diferentes áreas y disciplinas (Ruvalcaba-Coyaso et al. 2011). Balderas Gutiérrez (2013) conceptualiza a la identidad profesional como las representaciones que le permiten a un miembro de un grupo profesional describirse y compararse con otras profesiones. En este sentido, la identidad profesional hace referencia a los atributos, las características y las prácticas con las que un sujeto se identifica y vive como propias, como resultado de reconocerse como parte de un gremio o grupo profesional particular. Atendiendo a la relación existente entre las identidades profesionales y las prácticas de las personas en el marco de su trabajo, se hace evidente el interés del estudio de las identidades profesionales de los extensionistas rurales, como vía para comprender su desempeño profesional.

Reconstruyendo antecedentes, se observa que algunos autores han analizado las identidades de los extensionistas rurales; para Martín (2010), la identidad profesional de los extensionistas se encuentra organizada a partir de los principios del paradigma de la modernización, particularmente, la premisa de difusión de una racionalidad moderna y occidental apoyada en el positivismo, opuesta a la racionalidad cultural, propia de las comunidades locales. Por otra parte, Méndez Sastoque (2015) destaca que la identidad profesional de los extensionistas se construye en torno a la experiencia de trabajo con los productores y se caracteriza por un sentimiento de satisfacción, a partir del apoyo brindado a poblaciones vulnerables, mientras que Albaladejo et al. (2007) estudiaron la identidad de extensionistas rurales en Francia, diferenciando entre una identidad laboral, vinculada con las obligaciones institucionales y con la cultura organizacional de las entidades que los contratan y una identidad propiamente profesional, relacionada con sus prácticas en las comunidades, que van más allá de su rol institucional y de sus conocimientos académicos.

Se pueden identificar tres modos generales de comprender a las prácticas de extensión rural, que van a dar forma a tres identidades profesionales diferenciadas. Una primera perspectiva piensa a la extensión rural como una profesión orientada a la mejora de las prácticas productivas, comerciales u organizacionales de los productores. Este enfoque, va en línea con el modelo de extensión rural tradicional, surgido en América Latina, a mediados del siglo XX, que se orientaba a la transferencia o difusión de tecnologías modernas a los agricultores (Otero \& Selis, 2016). Si bien resulta discutible la persistencia de una extensión rural sostenida, estrictamente, en las mismas premisas, es claro la existencia de un enfoque que recorta el perfil del extensionista, como profesional focalizado en sus conocimientos técnico-productivos (Monsalvo Zamora et al. 2017). En esta línea, Landini (2016a), a partir de un estudio realizado en el noreste argentino, señala que los dos objetivos que se proponen los extensionistas rurales con mayor frecuencia son la mejora técnico-productiva de los agricultores y el fortalecimiento de sus organizaciones.

En paralelo, se puede observar una segunda forma de pensar a la extensión rural, que da forma a una identidad profesional, centrada en el aumento del bienestar y la calidad de vida de los productores. En esta línea, RELASER (2013) destaca la necesidad de una extensión rural que vaya más allá de las tecnologías agropecuarias y que contribuya a incrementar el capital social y disminuir los riesgos que enfrentan los pobres rurales. En particular, Méndez Sastoque (2006) señala que los extensionistas deben acompañar procesos de desarrollo, contribuyendo a la mejora de las condiciones de vida de los pobladores rurales.

Además, también se puede señalar una tercera identidad profesional, que delimita el rol del extensionista, como gestor de procesos de 
cambio social, orientados a la superación de situaciones de injusticia y de opresión social. Esta visión de la extensión rural, se apoya en los trabajos de Freire (1973), quien desde un enfoque humanista crítico discute las prácticas tradicionales de extensión rural, a las que considera como formas de imposición cultural. En general, esta tercera forma de pensar la identidad de los extensionistas, se asocia a un análisis de las problemáticas enfrentadas por los pequeños agricultores, en términos de relaciones de poder. Así, por ejemplo, se suele hacer referencia a problemas, como la expansión del agronegocio y la concentración de la tierra (Catullo et al. 2014), la implementación de modelos productivos que generan dependencia de empresas proveedoras de semillas y agroquímicos (Altieri, 2009) y la imposición de bajos precios de venta a los productores por parte de intermediarios y comercializadores (Caicedo Díaz, 2013).

Atendiendo a que las identidades profesionales de los extensionistas y los modelos de extensión rural predominantes en instituciones e, incluso, en países determinados se articulan con procesos sociohistórico-institucionales más amplios, cabe presentar algunas reflexiones. En primer lugar, se debe tener en cuenta que en las décadas del 80 y 90, del siglo XX, existió una fuerte presión privatizadora de los servicios de extensión rural, tanto en América Latina como a nivel mundial, en el marco de políticas neoliberales; por ejemplo, en Nueva Zelanda, se encuentra privatizado desde la década del 80 , en tanto que el sistema de extensión público de Australia, se encuentra en proceso de privatización (Diesel et al. 2008).

Por su parte, en la última década, se observan organizaciones internacionales que critican, tanto los modelos tradicionales difusionistas de extensión como la transferencia acrítica de esquemas privatizadores, a países con condiciones muy diferentes, a las de aquellos en los que estos modelos resultaron efectivos (Ardila, 2010). En esta línea, se destaca, cada vez más, la necesidad de esquemas de extensión pluralistas, que puedan dar una respuesta a las necesidades de diferentes tipos de productores, en el marco de procesos de articulación público-privados (Sulaiman \& Davis, 2012).

Finalmente, también se debe reconocer que los enfoques de extensión imperantes, se encuentran enmarcados en la discusión en torno a la contraposición entre modelos productivos convencionales, centrados en el paradigma de la revolución verde y propuestas de carácter agroecológico, que ponen en cuestión los sistemas agroalimentarios dominantes. En este contexto, se observa que, si bien el desarrollo de la agroecología ha sido heterogéneo, a nivel global, posee una fuerte presencia en América Latina (Gliessman, 2017), mientras que su adopción es menor en países industrializados, como Australia y Nueva Zelanda (Altieri et al. 2017) y posee poca presencia en Nigeria y Sudáfrica (Ogbalubi \& Wokocha, 2013).

En este marco, el presente artículo, se propone contribuir a la comprensión de las identidades profesionales de los extensionistas rurales en diversos países, a partir de la diferenciación entre tres identidades posibles, una centrada en el apoyo técnico-productivo, otra, orientada a la mejora de la calidad de vida de los productores y, otra, que piensa a la extensión rural en términos de lucha contra la desigualdad y la exclusión de los agricultores más pequeños.

\section{MATERIALES Y MÉTODOS}

En este artículo, se presentan y se analizan resultados de un cuestionario realizado a extensionistas rurales, entre 2017 y 2019, en nueve países: Argentina $(n=598)$, Australia $(n=35)$, Brasil ( $\mathrm{n}=265)$, Chile ( $\mathrm{n}=188)$, México $(\mathrm{n}=1509)$, Nueva Zelanda $(\mathrm{n}=17)$, Nigeria ( $n=93)$, Paraguay $(n=22)$ y Sudáfrica $(n=43)$. Estos países fueron seleccionados por la existencia de apoyo de instituciones o investigadores locales, dispuestos a facilitar el acceso a las muestras de extensionistas y por el interés de incluir países de diferentes continentes y regiones, a fin de aumentar la diversidad de respuestas.

El cuestionario forma parte de un estudio internacional, que tuvo por objeto conocer las creencias y las perspectivas de los extensionistas respecto de diferentes aspectos de su práctica, por lo que abordó temáticas que exceden el presente artículo. Los temas incluidos en el cuestionario fueron ajustados con el apoyo de doce expertos internacionales de Australia, Brasil, Estados Unidos, Francia, India, Nicaragua, Nigeria, Países Bajos, Pakistán, Sudáfrica. Con el objetivo de chequear la comprensión de las consignas del cuestionario, se realizó una prueba piloto en Argentina.

Los tamaños de muestra indicados corresponden a la cantidad de participantes que respondieron a la pregunta referida a la identidad profesional. El muestreo fue intencional y por conveniencia. Los participantes fueron contactados, con el apoyo de diferentes tipos de instituciones, las cuales, fueron seleccionadas por su importancia en los países respectivos y por la existencia de contactos locales, que hicieran posible el acceso. En Argentina, los encuestados trabajaban mayormente en el Instituto Nacional de Tecnología Agropecuaria y la Subsecretaría de Agricultura Familiar; en Brasil, en las Empresas Públicas de Asistencia Técnica y Extensión Rural (EMATER) de los estados de Amazonas, Pernambuco y Santa Catarina; en Chile, en el Instituto de Desarrollo Agropecuario y, en Paraguay, en la Dirección de Extensión Agraria. En Australia y Nueva Zelanda, los extensionistas fueron invitados por medio de la Red de Extensión de Australasia y el Pacífico (Australasia-Pacific Extension Network) y por medio del Instituto Neozelandés de Administración de la Industria Primaria (New Zealand Institute of Primary Industry Management). En México, se contactó con integrantes del listado público del Sistema de Extensionismo Rural Mexicano. Finalmente, en Nigeria y en Sudáfrica, los participantes fueron invitados por medio de dos organizaciones profesionales de nivel nacional, el Foro Nigeriano para los Servicios de Asesoramiento y la Extensión Agrícola (Nigerian Forum for Agricultural Extension and Advisory Services) y la Sociedad Sudafricana para la Extensión Agrícola (South African Society for Agricultural Extension). Las diferencias en los tamaños muestrales, se deben a las diferencias en las cantidades de extensionistas a los que se pudo acceder, a partir de las instituciones colaboradoras y del diferente grado de apoyo ofrecido por cada una de ellas.

En la mayor parte de los casos, el cuestionario fue enviado por correo electrónico y respondido en línea, por medio de la plataforma SurveyMonkey, con excepción de algunos casos en Sudáfrica, donde se recibieron algunas respuestas escaneadas. El cuestionario contenía 
preguntas sociodemográficas, como edad, género, nivel educativo y experiencia como extensionista, así como preguntas relativas a creencias sobre extensión e innovación, metodologías de extensión y enfoque productivo. El tiempo promedio de respuesta se estimó en media hora. En este artículo, se destaca la pregunta relativa a la identidad de los extensionistas, según la siguiente consigna:

A continuación, se presentan tres frases. Por favor, ordénelas de aquella con la que se siente más identificado/a, a nivel personal, a aquella con la que se siente menos. Use el número 1, para indicar aquella con la que más se identifica; 2 , para la siguiente y 3 , para aquella con la que se identifica menos. Tenga en cuenta que preguntamos por su opinión personal, no por la de la institución o empresa en la que trabaja:

- El extensionista rural es un trabajador o un profesional que ayuda a mejorar las prácticas productivas, comerciales u organizativas de los productores.

- El extensionista rural ayuda a los productores a aumentar su bienestar y mejorar su calidad de vida.

- El extensionista rural apoya a los productores para que puedan superar situaciones de injusticia y opresión.

La consigna llevó a la construcción de tres variables ordinales. Una referida a cada identidad profesional posible; en ellas, el valor de la variable va de 1 a 3 , según el orden de preferencia asignada a la alternativa. A la vez, estas tres variables fueron convertidas en una variable nominal, que indica cuál de las tres opciones fue elegida en primer lugar. Los resultados fueron procesados con el apoyo del software para análisis cuantitativo SPSS, atendiendo al nivel de medición de cada variable.

Junto a las tablas y figuras descriptivas, también se aprovechó la disponibilidad de información sociodemográfica y relativa a otras variables vinculadas con el trabajo de extensión para explorar la existencia de relaciones estadísticas entre ellas y la identidad con la que más se identifican los extensionistas. Atendiendo a que las relaciones podrían ser diferentes, según los países, se optó por trabajar de manera independiente, con las diferentes muestras nacionales.

Se incluyeron siete variables al análisis; cinco de ellas, variables sociodemográficas (edad, experiencia, género, nivel educativo y área de formación universitaria). Dada la existencia de diferencias en el modo de nombrar las carreras universitarias en los diferentes países, se optó por analizar esta variable solo en países latinoamericanos, ya que no se consideraron confiables los datos disponibles en idioma inglés. Adicionalmente, también se sumaron otras dos variables. Una de ellas, refiere al posicionamiento personal del extensionista, en una escala ordinal, en el marco del contraste 'agroecología' y 'agricultura convencional', siendo agroecología, 1 y agricultura convencional, 5 .

Por último, también se incorporó la variable 'grado de difusionismo', que refiere a una de las dimensiones de la Escala de Creencias sobre Extensión e Innovación, incluida en el cuestionario (Landini \& Beramendi, 2019). El 'grado de difusionismo' de un extensionista refiere al grado en que concibe a la extensión rural como transferencia de tecnologías de expertos a productores. La variable va de 1 a 5 , significando: 1 , total desacuerdo; 3 , ni de acuerdo ni en desacuerdo y 5 , total acuerdo, es decir, cuanto más difusionista sea un extensionista, tendrá puntajes más cercanos a 5 .

\section{RESULTADOS Y DISCUSIÓN}

Identidades profesionales seleccionadas como primera opción. Los resultados muestran que existen diferencias estadísticamente significativas entre los países, con relación a la identidad profesional $\left(\chi^{2}(12)=121 ; p<.001\right)$.

Analizando la media de los nueve países indagados (Tabla 1), se observa que la identidad más frecuente es la de un profesional orientado a la mejora productivo-empresarial de los productores agropecuarios. Este resultado es consistente, tanto con los principios de la revolución verde, que dieron impulso a una extensión rural orientada a la transferencia de tecnologías (Rogers, 1962), como con el perfil formativo de los extensionistas rurales, generalmente profesionales de las ciencias agropecuarias (Landini \& Bianqui, 2014). Al mismo tiempo, se debe tener presente que, hoy, el aumento de la producción, la productividad y las mejoras tecnológicas son objetivos centrales de numerosas iniciativas e instituciones de extensión rural (Méndez Sastoque, 2015; Landini, 2016a; Monsalvo Zamora et al. 2017).

En segundo lugar, se observa que poco más de un tercio de los extensionistas (en el promedio de los países), se identifica con un perfil profesional orientado al aumento del bienestar y la mejora de la calidad de vida de los productores. Si se piensa esta perspectiva identitaria desde las propuestas clásicas de difusión tecnológica, es esperable que, esta identidad profesional, se perciba como extraña; no obstante, resulta claramente consistente con los abordajes que conceptualizan a lo rural desde una perspectiva multidimensional y no solo agrícola (Méndez Sastoque, 2006) y aquellos que proponen pensar al extensionista no como un experto técnico sino como un agente de desarrollo rural (Ardila, 2010; Landini, 2016b).

Resulta interesante señalar que los países en los que se valoró más esta identidad (Argentina, Brasil, Chile y Paraguay) son latinoamericanos y se ubican geográficamente cercanos unos a otros, lo que podría hablar de la existencia de marcos institucionales compartidos y de experiencias formativas cruzadas entre ellos. En particular, se puede mencionar la creación en el 2004, de la Reunión Especializada sobre Agricultura Familiar del Mercado Común del Cono Sur (REAF MERCOSUR), iniciativa que focaliza en la agricultura familiar en el marco del bloque regional, conformado en el año de su creación, por Argentina, Brasil, Paraguay y Uruguay.

Por otra parte, cabe destacar el pequeño porcentaje de extensionistas que escogió la identidad asociada a la contribución con procesos de cambio social, a partir de la toma de conciencia de las relaciones de desigualdad, incluso opresión, en que se encuentran muchos pequeños agricultores. Esto resulta hasta cierto punto llamativo, teniendo en cuenta distintos señalamientos, que destacan problemas estructurales que enfrenta la pequeña agricultura, como la expansión 
Tabla 1. Identidades seleccionadas como primera opción según país.

\begin{tabular}{|c|c|c|c|}
\hline Países & Id: Mejora productivo-empresarial & Id: Mejora calidad de vida & Id: Cambio social \\
\hline Argentina & $47,6 \%{ }^{\mathrm{a}}$ & $47,7 \%{ }^{\mathrm{a}}$ & $4,7 \%{ }^{\mathrm{ab}}$ \\
\hline Australia & $91,4 \%{ }^{\mathrm{c}}$ & $8,6 \%{ }^{\mathrm{c}}$ & $0 \%{ }^{\mathrm{abc}}$ \\
\hline Brasil & $50,9^{\circ}{ }^{\mathrm{a}}$ & $44,5 \%{ }^{\mathrm{a}}$ & $4,5 \%$ abc \\
\hline Chile & $49,5 \%{ }^{\mathrm{a}}$ & $48,4 \%^{\mathrm{a}}$ & $2,1 \%{ }^{\mathrm{bc}}$ \\
\hline México & $67,0 \%{ }^{\mathrm{b}}$ & $30,1 \%{ }^{\mathrm{b}}$ & $2,9 \%{ }^{\mathrm{c}}$ \\
\hline Nigeria & $65,6 \%{ }^{\mathrm{b}}$ & $25,8 \%{ }^{\mathrm{b}}$ & $8,6 \%^{\mathrm{a}}$ \\
\hline $\begin{array}{l}\text { Nueva } \\
\text { Zelanda }\end{array}$ & $64,7 \%$ ab & $35,3 \%$ ab & $0 \%{ }^{\mathrm{abc}}$ \\
\hline Paraguay & $54,5 \%$ ab & $45,5 \%$ ab & $0 \%$ abc \\
\hline Sudáfrica & $58,1 \%{ }^{\mathrm{ab}}$ & $32,6 \%$ & $9,3 \%^{a}$ \\
\hline Promedio & $61 \%$ & $35,4 \%$ & $3,6 \%$ \\
\hline
\end{tabular}

Las letras en superíndice indican grupos de países, cuyos porcentajes referidos a cada columna no difieren significativamente entre ellos, a un nivel de $\mathrm{p}<0,05$ (se utilizó prueba $\mathrm{Z}$ sin Bonferroni).

del agronegocio, la concentración de la tierra, la implementación de modelos productivos que generan dependencia por parte de los agricultores y el rol dominante que ocupan los comercializadores en las cadenas agroindustriales (Altieri, 2009; Caicedo Díaz, 2013; Catullo et al. 2014); sin embargo, el resultado es consistente con un estudio empírico, efectuado en 10 países latinoamericanos, que muestra que, en general, los extensionistas rurales son poco críticos de los modelos de extensión y desarrollo rural que subyacen a sus prácticas (Landini, 2016c).

En este contexto, se observó que los dos países con un mayor porcentaje de extensionistas, con una identidad asociada a la idea de cambio social, son Nigeria, uno de los más pobres del mundo (Banco Mundial, 2020a) y Sudáfrica, uno de los más desiguales (Banco Mundial, 2020b). Esto podría sugerir que, las identidades asociadas a la superación de situaciones de injusticia y de opresión, podrían estar relacionadas con contextos nacionales, caracterizados por pobreza y por desigualdad extremas; no obstante, la realidad de desigualdad que vive gran parte de América Latina pone en cuestión esta posible interpretación.

A partir del análisis de la figura 1, se pueden identificar tres tipos de países. En el primer grupo, compuesto únicamente por Australia, predomina ampliamente una identidad profesional, asociada al apoyo productivo-empresarial a los agricultores; por su parte, también se observa un segundo grupo, conformado por México, Nigeria, Nueva Zelanda y Sudáfrica, donde se destaca una identidad profesional, centrada en el apoyo técnico-productivo, pero con presencia de extensionistas, quienes priorizan la mejora de la calidad de vida. Finalmente, existe un tercer grupo, donde la identidad profesional de apoyo productivo-empresarial cosecha, aproximadamente, el mismo nivel de preferencias, que la ayuda a mejorar la calidad de vida de los productores. Este grupo de países está compuesto por Argentina, Brasil, Chile y Paraguay, todos latinoamericanos.
Las razones de estas diferencias no son claras; sin embargo, tiene sentido el predominio de identidades asociadas al apoyo productivoempresarial en Australia y Nueva Zelanda, países con agricultura casi exclusivamente empresarial y sistemas de extensión privatizados o en proceso de privatización. Igualmente, los resultados de Argentina, Brasil, Chile y Paraguay, donde tiene mayor presencia la búsqueda de mejora de la calidad de vista de los productores, son consistentes con el mayor valor, dado a la dimensión social de la agricultura, expresado en la mayor presencia del movimiento agroecológico en América Latina (Gliessman, 2017).

Identidades profesionales por orden de preferencia según país. En el subtítulo anterior, se evaluaron las identidades profesionales seleccionadas, en primer lugar, en los diferentes países. En este apartado, se les analiza atendiendo el orden en que cada alternativa fue seleccionada por los extensionistas.

Utilizando el estadístico Kruskal Wallis, que permite comparar la distribución de variables ordinales en diferentes grupos, se observó que existen diferencias estadísticamente significativas entre los países, en el orden en que las alternativas identitarias fueron seleccionadas (identidad profesional orientada a la mejora productivo-empresarial: $\chi^{2}(8)=103, \mathrm{p}<.001$, identidad orientada a la mejora de la calidad de vida: $\chi^{2}{ }_{(8)}=85,5, \mathrm{p}<.001$ e identidad centrada en la superación de situaciones de injusticia y opresión: $\left.\chi^{2}(8)=35,3, \mathrm{p}<.001\right)$. Las distribuciones porcentuales de cada selección y las diferencias entre países son presentadas en las tablas 2, 3 y 4 .

A partir del análisis de la tabla 2, se observa que los extensionistas australianos y mexicanos, se destacan por priorizar una identidad profesional asociada a la mejora productivo-empresarial de los productores, en contraste con Chile, Brasil y Argentina, donde la alternativa posee menor relevancia comparativa, aun siendo ubicada como primera opción, en casi el 50\% de los casos. Estos resultados 


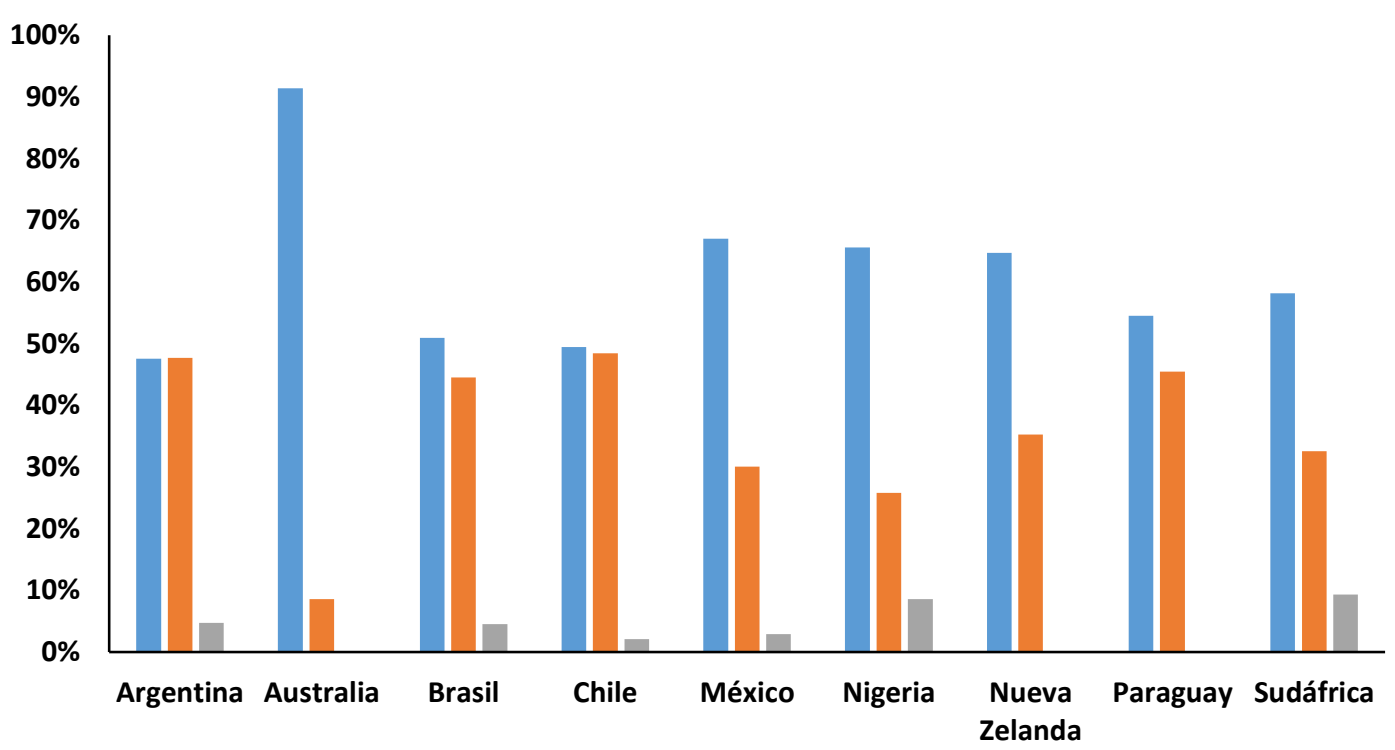

Id: Mejora productivo-empresarial

Id: Mejora calidad de vida

Id: Cambio social

Figura 1. Identidades seleccionadas como primera opción según país.

son consistentes con los del análisis previo, aunque evidencian con más claridad el carácter discordante de los resultados de México, país donde se esperaría más cercanía a los resultados de otros países de América Latina. Posiblemente, esto se deba a que la muestra mexicana no corresponde, propiamente, a un sistema de extensión público sino a un sistema de extensión tercerizado, financiado por el Estado, lo que se podría asociar a un perfil más cercano a la asistencia técnico-productiva, que a un apoyo más integral.

Por su parte, a partir de la tabla 3, se puede concluir que Chile, Argentina, Paraguay y Brasil, se caracterizan por un mayor porcentaje de extensionistas con identidades profesionales asociadas con la propuesta de apoyar a los productores, para que mejoren su calidad de vida. En contraste, se observa que en México, Nigeria y Australia esta identidad profesional es menos frecuente, todos resultados en línea, con los hallazgos analizados previamente.

Por último, la tabla 4, también permite reforzar conclusiones previas, observándose que, en Nigeria, Sudáfrica y Argentina, si bien es poco frecuente, los extensionistas adoptan más una identidad profesional asociada a la idea de cambio social, en contraste con lo que se observa en México, Australia, Chile y Paraguay. Este resultado es consistente con el estudio de Landini (2016c), sobre enfoques de extensión en América Latina, que muestra que, si bien de manera poco frecuente, en Argentina los extensionistas son más críticos de los modelos de desarrollo y extensión rural que en Brasil, Chile, México y Paraguay.

Variables asociadas a las distintas identidades profesionales. Se analizó la posible relación de diferentes variables con las identidades profesionales de los extensionistas. Como se observa en la tabla 5 , los resultados muestran que ninguna de las variables estudiadas se encuentra asociada de manera sistemática con las identidades profesionales de los extensionistas rurales, en los diferentes países.

Realizando un análisis más detallado, se observa que los resultados no evidencian relaciones estadísticamente significativas en el caso de la edad y el área de formación (título universitario), en ninguno de los países. Esto resulta llamativo, en tanto hubiera sido esperable que los extensionistas ingenieros agrónomos o veterinarios tuvieran, por ejemplo, una identidad más cercana al apoyo técnico productivo y aquellos formados en ciencias sociales, una identidad orientada a la mejora de la calidad de vida de los productores o, incluso, al impulso de procesos de cambio social.

En cuanto a las variables experiencia como extensionista y nivel educativo (variables potencialmente asociadas entre sí a partir de la edad), en México, se observó que, mientras que quienes poseen menos experiencia y formación, tienden a identificarse con la idea de contribuir a la mejora productivo-empresarial de los productores; quienes poseen más años en la profesión y mayor nivel educativo, se identifican con mayor frecuencia con la propuesta de mejora de la calidad de vida de los productores. A partir de estas relaciones, se podría pensar que, tanto la experiencia como la mayor formación, permiten a los extensionistas tomar conciencia que los productores no solo necesitan mejoras tecnológico-empresariales, sino también otros apoyos vinculados con la mejora de la calidad de vida.

Respecto de la variable género, los resultados obtenidos en Argentina sugieren que la idea de apoyar a los agricultores, a nivel productivo y empresarial, es más frecuente en los hombres, mientras que las mujeres suelen pensar a la extensión con más frecuencia, como una labor orientada a superar situaciones de injusticia y opresión. Si bien las razones de estos resultados no son claras, se podría pensar que 
Tabla 2. Identidad extensionista orientada a la mejora productivo-empresarial según país.

\begin{tabular}{|c|c|c|c|c|}
\hline \multirow{2}{*}{ Países } & \multirow{2}{*}{ Rango medio } & \multicolumn{3}{|c|}{ Orden de priorización } \\
\hline & & 1 & 2 & 3 \\
\hline Australia $^{a}$ & 958 & $91,4 \%$ & $5,7 \%$ & $2,9 \%$ \\
\hline México $^{b}$ & 1285 & $67 \%$ & $28,1 \%$ & $4,9 \%$ \\
\hline Nueva Zeland $\mathrm{a}^{\mathrm{bc}}$ & 1320 & $65,6 \%$ & $24,7 \%$ & $9,7 \%$ \\
\hline Nigeria $^{\text {bc }}$ & 1330 & $64,7 \%$ & $29,4 \%$ & $5,9 \%$ \\
\hline Paraguay $^{\text {bc }}$ & 1419 & $54,5 \%$ & $45,5 \%$ & $0 \%$ \\
\hline Sudáfrica $^{\mathrm{bc}}$ & 1448 & $58,1 \%$ & $27,9 \%$ & $14 \%$ \\
\hline Chile $^{c}$ & 1508 & $49,5 \%$ & $46,3 \%$ & $4,3 \%$ \\
\hline Brasil $^{c}$ & 1493 & $50,9 \%$ & $44,2 \%$ & $4,9 \%$ \\
\hline Argentina $^{c}$ & 1573 & $47,6 \%$ & $41,0 \%$ & $11,5 \%$ \\
\hline \multicolumn{2}{|c|}{ Promedio } & $61 \%$ & $32,5 \%$ & $6,5 \%$ \\
\hline
\end{tabular}

Las letras en superíndice indican grupos de países que difieren significativamente entre ellos, en términos de distribución de la variable, a un nivel de $\mathrm{p}<0,05$.

Tabla 3. Identidad extensionista orientada a la mejora de la calidad de vida según país.

\begin{tabular}{|l|c|c|c|c|}
\hline \multirow{2}{*}{ Países } & \multirow{2}{*}{ Rango medio } & \multicolumn{3}{|c|}{ Orden de priorización } \\
\cline { 3 - 5 } & & $\mathbf{1}$ & $\mathbf{2}$ & $\mathbf{3}$ \\
\hline Chile $^{\mathrm{a}}$ & 1198 & $48,4 \%$ & $48,9 \%$ & $2,7 \%$ \\
\hline Argentina $^{\mathrm{a}}$ & 1230 & $47,7 \%$ & $47 \%$ & $5,2 \%$ \\
\hline Paraguay $^{\mathrm{a}}$ & 1253 & $45,5 \%$ & $50 \%$ & $4,5 \%$ \\
\hline Brasil $^{\mathrm{a}}$ & 1279 & $44,5 \%$ & $49,4 \%$ & $6 \%$ \\
\hline Nueva Zelanda $^{\text {ab }}$ & 1398 & $35,3 \%$ & $58,8 \%$ & $5,9 \%$ \\
\hline Sudáfrica $^{\text {ab }}$ & 1422 & $32,6 \%$ & $62,8 \%$ & $4,7 \%$ \\
\hline México $^{\mathrm{b}}$ & 1464 & $30,1 \%$ & $64,2 \%$ & $5,7 \%$ \\
\hline Nigeria $^{\text {bc }}$ & 1593 & $25,8 \%$ & $60,2 \%$ & $14 \%$ \\
\hline Australia $^{\mathrm{c}}$ & 1745 & $8,6 \%$ & $85,7 \%$ & $5,7 \%$ \\
\hline \multicolumn{2}{|c|}{ Promedio } & $35,4 \%$ & $58,6 \%$ & $6 \%$ \\
\hline
\end{tabular}

Las letras en superíndice indican grupos de países que difieren significativamente entre ellos, en términos de distribución de la variable, a un nivel de $\mathrm{p}<0,05$.

Tabla 4. Identidad extensionista orientada al cambio social según país.

\begin{tabular}{|c|c|c|c|c|}
\hline \multirow{2}{*}{ Países } & \multirow{2}{*}{ Rango medio } & \multicolumn{3}{|c|}{ Orden de priorización } \\
\hline & & 1 & 2 & 3 \\
\hline Nigeria $^{a}$ & 1222 & $8,6 \%$ & $15,1 \%$ & $76,3 \%$ \\
\hline South Africa ${ }^{\text {ab }}$ & 1288 & $9,3 \%$ & $9,3 \%$ & $81,4 \%$ \\
\hline Argentina $^{\text {ab }}$ & 1321 & $4,7 \%$ & $12 \%$ & $83,3 \%$ \\
\hline New Zealanda ${ }^{\text {bc }}$ & 1395 & $0 \%$ & $11,8 \%$ & $88,2 \%$ \\
\hline Brasil $^{\text {bc }}$ & 1398 & $4,5 \%$ & $6,4 \%$ & $89,1 \%$ \\
\hline Mexico $^{c}$ & 1405 & $2,9 \%$ & $7,7 \%$ & $89,4 \%$ \\
\hline Australia $^{\mathrm{c}}$ & 1437 & $0 \%$ & $8,6 \%$ & $91,4 \%$ \\
\hline Chile $^{c}$ & 1456 & $2,1 \%$ & $4,8 \%$ & $93,1 \%$ \\
\hline Paraguay $^{\mathrm{c}}$ & 1491 & $0 \%$ & $4,5 \%$ & $95,5 \%$ \\
\hline \multicolumn{2}{|c|}{ Promedio } & $3,6 \%$ & $8,9 \%$ & $87,5 \%$ \\
\hline
\end{tabular}

Las letras en superíndice indican grupos de países que difieren significativamente entre ellos, en términos de distribución de la variable, a un nivel de p<0,05. 
Tabla 5. Variables asociadas a las identidades profesionales en diferentes países.

\begin{tabular}{|c|c|c|c|c|c|c|c|}
\hline & Edad & $\begin{array}{c}\text { Experiencia } \\
\text { (en años) }\end{array}$ & Sexo & Nivel educativo & $\begin{array}{l}\text { Área de } \\
\text { formación }\end{array}$ & $\begin{array}{c}\text { Valoración agro- } \\
\text { ecología }\end{array}$ & $\begin{array}{l}\text { Grado de difu- } \\
\text { sionismo }\end{array}$ \\
\hline Argentina & $\mathrm{F}_{(2,590)}=1,09$ & $\mathrm{~F}_{(2,590)}=0,88$ & $\begin{array}{c}X^{2}(2)=7,68^{*} \\
\mathrm{Id}_{1}=\mathrm{H}: 70,2 \% \\
\mathrm{Id}_{2}=\mathrm{H}: 61,5 \% \\
\mathrm{Id}_{3}=\mathrm{H}: 50 \%\end{array}$ & $\mathrm{KW}: \chi^{\mathbf{2}(2)}=2,13$ & $X^{2}{ }_{(10)}=15,62$ & $\begin{aligned} K W: X_{(2)}^{2} & =8,66^{*} \\
\mathrm{RM} \mathrm{Id}_{1} & =303^{\mathrm{a}} \\
\mathrm{RM} \mathrm{Id}_{2} & =289^{\mathrm{a}} \\
\mathrm{RM} \mathrm{Id}_{3} & =209^{\mathrm{b}}\end{aligned}$ & $\begin{aligned} \mathrm{F}_{(2,590)} & =23,8^{* *} \\
\mathrm{Id}_{1} & =3,14^{\mathrm{a}} \\
\mathrm{Id}_{2} & =2,87^{\mathrm{b}} \\
\mathrm{Id}_{3} & =2,38^{\mathrm{c}}\end{aligned}$ \\
\hline Australia & $t_{(33)}=-0,92$ & $\mathrm{t}(33)=0,10$ & $X^{2}(1)=0,43$ & MW: $U=67$ & Sin datos & MW: $U=33,5$ & $\mathrm{t}(33)=0,32$ \\
\hline Brasil & $F_{(2,262)}=1,06$ & $\mathrm{~F}_{(2,262)}=1,89$ & $X^{2}(2)=0,58$ & $\mathrm{KW}: \chi^{2}(2)=2,80$ & $X^{2}{ }_{(10)}=7,41$ & $\mathrm{KW}: \chi^{2}(2)=0,73$ & $F_{(2,262)}=1,87$ \\
\hline Chile & $\mathrm{F}_{(2,185)}=0,28$ & $\mathrm{~F}_{(2,185)}=0,65$ & $X^{2}(2)=1,16$ & $\mathrm{KW}: \chi_{(2)}^{2}=2,79$ & $X^{2}(10)=15,21$ & $\mathrm{KW}: \chi^{2}(2)=0,50$ & $\begin{array}{c}\mathrm{F}_{(2,185)}=11,1^{* *} \\
\mathrm{Id}_{1}=3,43^{\mathrm{a}} \\
\mathrm{Id}_{2}=3,07^{\mathrm{b}} \\
\mathrm{Id}_{3}=4,17^{\mathrm{a}}\end{array}$ \\
\hline México & $F_{(2,1505)}=2,28$ & $\begin{array}{c}\mathrm{F}_{(2,1505)}=6,48^{* *} \\
\mathrm{Id}_{1}=8,20^{\mathrm{a}} \\
\mathrm{Id}_{2}=9,94^{\mathrm{b}} \\
\mathrm{Id}_{3}=8,23^{\mathrm{ab}}\end{array}$ & $\mathbf{X}^{2}(2)=1,08$ & $\begin{aligned} K W: X^{2}{ }_{(2)} & =7,46^{*} \\
\mathrm{RM} \mathrm{Id}_{1} & =731^{\mathrm{a}} \\
\mathrm{RM} \mathrm{Id}_{2} & =778^{\mathrm{b}} \\
\mathrm{RMId}_{3} & =735^{\mathrm{ab}}\end{aligned}$ & $X^{2}(10)=13,37$ & $\begin{aligned} \mathrm{KW}: \mathrm{X}^{2}(2) & =6,63^{*} \\
\mathrm{RM} \mathrm{Id}_{1} & =761^{\mathrm{a}} \\
\mathrm{RM} \mathrm{Id}_{2} & =709^{\mathrm{a}} \\
\mathrm{RM} \mathrm{Id}_{3} & =661^{\mathrm{a}}\end{aligned}$ & $\begin{array}{c}\mathrm{F}_{(2,1505)}=6,24^{* *} \\
\mathrm{Id}_{1}=3,75^{\mathrm{a}} \\
\mathrm{Id}_{2}=3,60^{\mathrm{b}} \\
\mathrm{Id}_{3}=3,64^{\mathrm{ab}}\end{array}$ \\
\hline Nigeria & $F_{(2,90)}=0,78$ & $F_{(2,90)}=1,20$ & $X^{2}(2)=2,85$ & $\mathrm{KW}: \chi^{2}(2)=2,37$ & Sin datos & $\mathrm{KW}: \chi^{2}{ }_{(2)}=1,19$ & $F_{(2,90)}=0,83$ \\
\hline $\begin{array}{c}\text { Nueva Ze- } \\
\text { landa }\end{array}$ & MW: $\mathrm{U}=43,5$ & MW: $\mathrm{U}=48,5$ & $X^{2}(2)=1,99$ & MW: $\mathrm{U}=30$ & Sin datos & MW: $U=29,5$ & MW: $U=23$ \\
\hline Paraguay & MW: $\mathrm{U}=40$ & MW: $U=44$ & $X^{2}(1)=0,63$ & MW: $\mathrm{U}=50$ & $X^{2}(4)=4,34$ & MW: $U=80,5$ & MW: $U=67,5$ \\
\hline Sudáfrica & $F_{(2,40)}=0,71$ & $F_{(2,39)}=2,52$ & $X^{2}(2)=2,15$ & $\mathrm{KW}: \chi^{2}(2)=4,01$ & Sin datos & $\mathrm{KW}: \chi_{(2)}^{2}=2,23$ & $F_{(2,40)}=1,07$ \\
\hline
\end{tabular}

${ }^{*} \mathrm{p}<0,05,{ }^{* *} \mathrm{p}<0,01$. Para nuestras menores se utilizan estadísticos no paramétricos. MW: U de Mann Whitney; KW: Kruskal Wallis; RM: Rango medio; Id1: Identidad 'Mejora productivo-empresarial'; Id2: Identidad de 'Mejora de calidad de vida'; Id3: Identidad 'Cambio social'. Cuando en un país no surgió la Id3, como primera alternativa, se utilizan los estadísticos correspondientes. Las letras en superíndice indican grupos que difieren significativamente entre ellos, a nivel estadístico $\mathrm{p}<.05$.

los hombres tienen un perfil de extensión más tradicional, centrado en las mejoras productivas, en tanto que las mujeres presentan, con más frecuencia, una mirada alternativa que cuestiona miradas hegemónicas.

Por otra parte, tanto en el caso de Argentina como en el de México, se observó la existencia de relación entre las identidades profesionales de los extensionistas y su orientación productiva. En particular, se encontró que, en ambos países, los extensionistas que plantean su trabajo en términos de procesos de cambio social y apoyo a la superación de situaciones de injusticia, suelen tener una posición más agroecológica que el resto, lo que resulta razonable si se tiene en cuenta la crítica de la agroecología a las relaciones de poder que se establecen en el marco del sistema agroalimentario, en detrimento de los pequeños productores (Altieri, 2009); no obstante, la comparación entre pares de identidades solo es significativa en Argentina, no en México.

Por último, la tabla 5, también evidenció la existencia de relaciones entre la identidad profesional de los extensionistas y su grado de acuerdo con una extensión rural difusionista, en este caso, en tres países: Argentina, Chile y México. En general, estos resultados muestran que aquellos que plantean su identidad, en términos de apoyo productivo-empresarial, tienden a pensar su trabajo más en términos de difusión de tecnologías que aquellos que se identifican con la idea de apoyo a calidad de vida de los productores. Esto parece resultar razonable, en tanto, son los primeros los más preocupados por la 'mejora tecnológica' de los productores, en tanto que los segundos, potencialmente, deben trabajar con relación a múltiples aspectos no técnico-productivos. Finalmente, llama la atención que, mientras que quienes construyen su identidad profesional como extensionistas en torno a la idea de superación de situaciones de desigualdad y opresión en Argentina son los menos difusionistas, mientras que, en Chile, son los que puntúan más alto en difusionismo, lo que invita a pensar en una relación compleja entre ambas variables. Así, en Argentina parecería pensarse al cambio social desde una perspectiva dialógica, participativa y construida horizontalmente, en tanto que, en Chile, desde una mirada más vertical, donde se ve necesario convencer a los productores de los determinantes sociales de las relaciones de desigualdad.

Limitaciones y recomendaciones para futuras investigaciones. En cuanto a las limitaciones del estudio, se debe tener en cuenta la existencia de diferentes tamaños en las muestras utilizadas, lo que invita a tomar con cautela los resultados de aquellos países con muestras más pequeñas. Por otra parte, se reconoce el interés de llevar adelante estudios cualitativos, que permitan complementar los resultados del presente trabajo, a fin de generar descripciones identitarias, que recojan la diversidad de formas en que los extensionistas rurales se comprenden a sí mismos 
y a sus prácticas profesionales. Incluso, en futuros trabajos, se podría pensar la integración de preguntas cerradas y abiertas, para permitir una expresión más flexible de los participantes. A la vez, en trabajos cualitativos futuros, también se podría indagar cuáles son las características identitarias más destacadas de un 'extensionista rural', desde la perspectiva de las personas entrevistadas y cómo se relacionan estas identidades con los conocimientos, afectos y valores de los extensionistas.

$\mathrm{El}$ presente trabajo, se propuso estudiar las identidades profesionales de los extensionistas rurales como forma de contribuir a la comprensión de sus prácticas. Los resultados obtenidos permiten concluir que la identidad profesional de los extensionistas rurales es diferente entre países e, incluso, al interior de estos según diferentes variables. La identidad más frecuente asocia la práctica de la extensión rural a la mejora productivo-empresarial de los productores, seguida por aquella que prioriza la búsqueda de una mejora en la calidad de vida. En tercer lugar y con mucha distancia, se observa un pequeño porcentaje de extensionistas que piensan su profesión en términos de impulsar procesos de cambio social a favor de los pequeños agricultores. A nivel general, se puede pensar en dos tipos de países: uno, donde la identidad orientada a la mejora técnico-productiva es mayoritaria y, otro, donde ambas identidades poseen porcentajes de adhesión similares.

Conflicto de intereses: El manuscrito fue preparado y revisado con la participación de todos los autores, quienes declaramos que no existe ningún conflicto de intereses que ponga en riesgo la validez de los resultados presentados. Financiación: Universidad de la Cuenca del Plata, Argentina y AgResearch, Nueva Zelanda.

\section{REFERENCIAS}

1. ALBALADEJO, C.; COUIX, N.; BARTHE, L. 2007. Learning in agriculture: rural development agents in France caught between a job identity and a professional identity. Journal of Agricultural Education and Extension (Holanda). 13(2):95-106. https://doi.org/10.1080/13892240701289361

2. ALTIERI, M. 2009. Agroecología, pequeñas fincas y soberanía alimentaria. Revista Ecología Política (España). 38:25-35.

3. Altieri, M.; NICHOLLS, C.; MONTALBA, R. 2017. Technological approaches to sustainable agriculture at a crossroads: An agroecological perspective. Sustainability. $9(3): 349$.

https://doi.org/10.3390/su9030349

4. ARDILA, J. 2010. La extensión rural para el desarrollo de la agricultura y la seguridad alimentaria. Aspectos conceptuales, situación y una visión de futuro. IICA. (Costa Rica). Disponible desde Internet en: http://repiica.iica.int/docs/B1898e/B1898e.pdf (con acceso 08/04/2021)
5. BALDERAS GUTIÉRREZ, K. 2013. Elementos que constituyen la identidad profesional de la enfermera. Atlante: Cuadernos de Educación y Desarrollo (España). $37 \mathrm{p}$.

6. BANCO MUNDIAL. 2020a. Poverty \& Equity Brief Nigeria Sub-Saharan Africa, Banco Mundial. Disponible desde Internet en:

https:/ / databank.worldbank.org/data/download/ poverty/33EF03BB-9722-4AE2-ABC7-AA2972D68AFE/ Global_POVEQ_NGA.pdf (con acceso 30/04/2020).

7. BANCO MUNDIAL. 2020b. Índice de Gini (USA). Disponible desde Internet en: https://datos.bancomundial.org/indicador/SI.POV.GINI (con acceso 30/04/2020).

8. CAICEDO DÍAZ, J.F. 2013. La intermediación como un impedimento al desarrollo del pequeño productor de Medellín. Corpoica (Colombia). 14(1):27-32. https://doi.org/10.21930/rcta.vol14_num1_art:264

9. CATUlLO, J.; VARELA, H.; ALEMANY, C.; TORRES, G.; CHÁVEZ, F.; BRUNEL, L.; BALSADI, O. 2014. Rol de la extensión rural en la gestión de innovaciones (Uruguay). Documento de PROCISUR.

10. DIESEL, V.; FROEHLICH, J.; NEUMANN, P.; SILVEIRA, P. 2008. "Privatização dos serviços de extensão rural: uma discussão (des)necessária? Revista de Economia e Sociologia Rural (Brasil). 46(4):1155-1188. https://doi.org/10.1590/S0103-20032008000400010

11. FREIRE, P. 1973. ¿Extensión o comunicación? La concientización en el medio rural. Ed. Siglo XXI (Buenos Aires, Argentina) 53p.

12. GLIESSMAN, S. 2017. A brief history of agroecology in Spain and Latin America. Agroecology and Sustainable Food Systems. 41(3-4):229-230. https://doi.org/10.1080/21683565.2017.1292390

13. LANDINI, F. 2016a. Enfoques y prácticas de extensión rural públicas en el noreste argentino. Revista de Economia e Sociologia Rural (Brasil). 54(1):167-186. http://doi.org/10.1590/1234-56781806-9479005401009

14. LANDINI, F. 2016b. How to be a good rural extensionist. Reflections and contributions of Argentine practitioners. Journal of Rural Studies (Reino Unido). 43(1):193-202. https://doi.org/10.1016/j.jrurstud.2015.11.014

15. LANDINI, F. 2016c. Concepción de extensión rural en 10 países latinoamericanos. Andamios (México). 13(30):211-236. 
16. LANDINI, F; BERAMENDI, M. 2019. Construction and validation of a psychometric scale to assess extension agents' beliefs about extension and innovation. The Journal of Agricultural Education and Extension (Países Bajos). 25(5):381-399.

https://doi.org/10.1080/1389224X.2019.1643748

17. LANDINI, F.; BIANQUI, V. 2014. Socio-demographic profile of different samples of Latin American rural extensionists. Ciência Rural (Brasil). 44(3):575-581. https://doi.org/10.1590/S0103-84782014000300030

18. MARTÍN, F. 2010. Ingenieros agrónomos, desarrollo rural y cultura política: reflexiones críticas en torno a la práctica de la (ex)tensión rural. Revista de la Facultad de Ciencias Agrarias UNCuyo (Argentina). 42(2):99-109.

19. MÉNDEZ SASTOQUE, J.M. 2006. Los retos de la extensión ante una nueva y cambiante noción de lo rural. Revista Facultad Nacional de Agronomía Medellín (Colombia). 59(2):3407-3423.

20. MÉNDEZ SASTOQUE, M.J. 2015. Satisfacción con el empleo de extensionista rural: un estudio cualitativo en Caldas, Colombia. Revista Ceres (Brasil). 62(3):241-250. https://doi.org/10.1590/0034-737X201562030003

21. MONSALVO ZAMORA, A.; JIMÉNEZ VELÁZQUEZ, M.; GARCÍA CUÉ, J.; SANGERMAN-JARQUÍN, D.; MARTÍNEZ SALDAÑA, T.; PIMENTEL EQUIHUA, J. 2017. Caracterización del perfil del extensionista rural en la zona oriente del Estado de México. Revista Mexicana de Ciencias Agrícolas. 8(3):503-515.

https://doi.org/10.29312/remexca.v8i3.27
22. OGBALUBI, L.; WOKOCHA, C. 2013. Agricultural development and employment generation: The Nigeria experience. IOSR Journal of Agriculture and Veterinary Science. 2(2):60-69.

23. OTERO, J.; SELIS, D. 2016. La Revista "Extensión en las Américas". Influencia de los EEUU en los servicios de extensión rural latinoamericanos. Extensão Rural (Brasil). 23(1):42-57.

http://dx.doi.org/10.5902/2318179617359

24. RED LATINOAMERICANA PARA SERVICIOS DE EXTENSIÓN RURAL, RELASER. 2013. Extensión rural con enfoque para la inclusión y el desarrollo rural Revista Claridades Agropecuarias (México). 243:42-48.

25. ROGERS, E. 1962. Diffusion of innovations. Ed. The Free Press (EEUU). 236p.

26. RUVALCABA-COYASO, J.; ALVARADO, I.; GARCÍA, R. 2011. Identidad e identidad profesional: Acercamiento conceptual e investigación contemporánea. CES Psicología (Colombia). 4(2):82-102. https://doi.org/10.21615/1254

27. SULAIMAN, R.; DAVIS, K. 2012. The "new extensionist": Roles, strategies, and capacities to strengthen extension and advisory services. GFRAS (Suiza). Disponible desde Internet en: https://www.g-fras.org/es/conocimientos/publicacionesdel-gfras.html?download=126:the-new-extensionist position-paper (con acceso 08/04/2021). 